Editorial

\title{
Foreword to the Special Section on the International Conference on E-Learning and
}

\section{Games 2016 (Edutainment '16)}

The International Conference on E-Learning and Games, or Edutainment in short, was inaugurated in 2006. Since then, the conference has been held in many places including Hong Kong (2007), Nanjing (China, 2008), Banff (Canada, 2009), Changchun (China, 2010), Taiwan (2011), etc. The conference covers not only game-based learning, but also learning experiences which may be gained from entertainment. The series provides an international forum for researchers and practitioners in various disciplines to share and exchange of experiences in the emerging research area combining education and entertainment.

This year (2016) the conference was held on $14^{\text {th }} \sim 16^{\text {th }}$ April, in Hangzhou, which is one of the most scenic cities in China. The conference has received about 50 submissions. With the help of reviewers from our International Program Committee, in the end 30 papers were accepted for presentation at the conference, covering a very wide range of topics, including E-learning, digital culture heritage, computer games, computer graphics and image processing. Based on the highest marks three contributions were invited to submit extended and augmented versions of their conference papers to Computers and Graphics. Through a highly competitive and selective review process according to the journal's criteria, only one paper has made it to this special section.

The paper, Real-Time Edge-Aware Weighted Median Filtering on the GPU, by Hanli Zhao, et al. [1], proposes a new 4D bilateral grid by incorporating the 3D bilateral grid with an additional range dimension. The edge-aware weights and the weighted median values are computed in 4D space. The proposed algorithm is highly parallel, enabling real-time GPU-based edge-aware implementation.

We are grateful to Prof. Joaquim Jorge, Ross E. Laman, Qian Jiao and all of the C\&G journal staff for facilitating the publication of this special section. We would like to thank the authors and the reviewers who were especially helpful with reviewing the manuscripts, for contributing to the success of the special section.

\section{References}

[1] Hanli Zhao, Dandan Gao, Ming Wang, and Zhigeng Pan, Real-Time Edge-Aware Weighted Median Filtering on the GPU, Comput. Graph. 2016

\section{Bios}

Feng Tian received the Ph.D. degree in Mechanical Engineering from Xi'an Jiaotong University, Xi'an, China in 1997. He is currently an Associate Professor of Media Technology in Bournemouth University, Bournemouth, U.K. He was an Assistant Professor in Nanyang Technological University in Singapore. His current research interests include computer graphics, computer animation, games technology, augmented reality, image processing.

Minghui Sun is a lecturer at Jilin University. He is a member of CCF, ACM and a committee member of ACM SIGCHI China Chapter. He is interested in using $\mathrm{HCl}$ methods to solve challenging real world computing problems in many areas, including multimodal interface (tactile modality), pen-based interface, tangible interface and user performance modelling. He was a Postdoctoral Fellowship in Riken, Japan and Dresden University of Technology, Germany. 